\title{
Cochrane review summary: pharmacological interventions for promoting smoking cessation during pregnancy
}

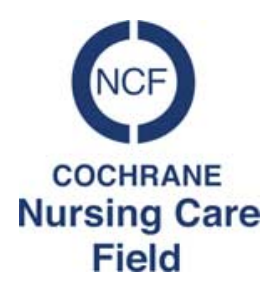

\section{Daksha Trivedi}

Senior Research Fellow, Evidence Based Practice, Centre for Research in Primary and Community Care, University of Hertfordshire, Hatfield, UK

Key words: drugs; interventions; pharmacotherapy; pregnancy; smoking cessation

Received 23 July 2013; accepted 24 July 2013

\section{Review question}

1. To determine whether any smoking cessation pharmacotherapies, including nicotine replacement therapy (NRT), varenicline and bupropion when used to support smoking cessation in pregnancy:

- are effective for smoking cessation in later pregnancy,

- affect infants' mean birth weights,

- affect rates of adverse perinatal outcomes.

2. To document from reports:

- adherence to treatment,

- minor adverse events,

- long-terms effects of smoking cessation pharmacotherapies.

\section{Relevance to primary care and nursing}

Primary care teams, including nurses, midwives and health visitors deliver advice on smoking cessation to pregnant women. The National Institute for Health and Clinical Excellence (NICE) has issued a guidance report for health care professionals on cautious use of NRT, during pregnancy although use of other pharmacotherapies during pregnancy is not currently recommended (NICE, 2010). This systematic review has summarised

Correspondence to: Dr Daksha Trivedi, Senior Research Fellow, Evidence Based Practice, Centre for Research in Primary and Community Care, University of Hertfordshire, College Lane, Hatfield AL10 9AB, UK. Email: d.trivedi@herts.ac.uk

C) Cambridge University Press 2013 all the available evidence for pharmacotherapies including NRT prescribed to support smoking cessation in pregnancy (Coleman et al., 2012).

\section{Type of review}

This is a summary of a Cochrane review containing six randomised controlled trials (RCTs). Data were combined in a meta-analysis, where appropriate.

\section{Characteristics of the evidence}

The review included a total of 1745 participants who were pregnant and current smokers (not defined) aged 16 years and over. The included studies comprised of women with gestation ages between 20 and 28 weeks in one study and at or after 32 weeks in the others. Two studies were conducted in the USA and the rest were from Australia, Canada, Denmark and England.

Interventions needed to be any pharmacological intervention (including NRT, varenicline and bupropion), aimed at stopping smoking in pregnant women with or without additional behavioural support (not defined) or cognitive behaviour therapy (not defined) or brief advice. There were no limitations to dosages, duration or type (eg, gum, patch, etc.). Trials must have provided very similar (ideally identical) levels of behavioural support or cognitive behaviour therapy (CBT) to participants in both the active drug and comparison groups. 
The primary efficacy outcome was smoking cessation in later pregnancy, measured at the latest point by self-report and validated biochemically from exhaled carbon monoxide or saliva/serum cotinine. Secondary outcomes included safety [assessed by seven birth outcomes of neonatal well-being: miscarriage/spontaneous abortion, stillbirth, mean adjusted birth weight, low birth weight $(<2500 \mathrm{~g})$, preterm birth ( $<37$ weeks' gestation), neonatal intensive care unit admissions, neonatal death, caesarean section)], adherence, non-serious adverse events and long-term effects of smoking cessation pharmacotherapies.

None of the included trials utilised varenicline or bupropion. NRT was provided as an adjunct to behavioural support and compared with behavioural support alone or support plus a placebo. Of the six included studies, four were placebo-RCTs (defined as any form of NRT or other pharmacotherapy with or without behavioural support/ cognitive behavioural therapy or brief advice compared with placebo NRT and additional support of similar intensity) and two compared NRT plus behavioural support with behavioural support alone. They were delivered during antenatal and prenatal visits by trained health professionals, including midwives.

The type and amount of NRT provided varied between studies. One study used $2 \mathrm{mg}$ nicotine gum and all other studies used nicotine patches. In one of these studies, one third of participants who were offered a choice of NRT preparations chose to use gum and lozenge. Four studies used $15 \mathrm{mg} / 16 \mathrm{~h}$. nicotine patches and one study used a higher nicotine dose $(21 \mathrm{mg} / 16 \mathrm{~h}$.) for participants who reported smoking more than 15 daily cigarettes. The current smokers reported smoking from $\geq 1$ to $\geq 15$ cigarettes a day.

Follow-up times varied from 1 week to three months from intervention and up to three months to a year postpartum. Validation of self-report was done within a few days of delivery, at final antenatal visit or four weeks before delivery. Point prevalence for self-report abstinence varied and included all time points, at 32-38 weeks, or at four weeks before delivery.

\section{Summary of key evidence}

Four studies were graded as high quality (low risk of bias) and two as low quality (high risk of bias).
The effects of interventions were analysed according to primary outcomes (efficacy) and secondary outcomes (safety). Data were pooled using risk ratios (RR) with $95 \%$ confidence interval (CI). The total number of studies and participants $(n)$ are shown in parenthesis. Effect sizes [mean differences (MD) or RR)] are also shown for studies that did not pool data.

\section{Primary outcome (efficacy)}

There was no statistically significant evidence that NRT was effective when compared with placebo/ control for smoking cessation in pregnancy (six studies, $n=1745$ ) (RR 1.33, 95\% CI 0.93-1.91). Sensitivity analysis using biochemically validated data rather than self-report data showed similar results.

\section{Secondary outcomes (safety)}

\section{Miscarriage/spontaneous abortion}

There was no statistically significant difference in risk of miscarriage/spontaneous abortion (three studies, $n=1407$ ) (RR 1.24, 95\% CI 0.37-4.17) or the numbers of stillbirths (three studies, $n=1402$ ) (RR 1.98 95\% CI 0.55-7.07) between NRT and control groups.

\section{Birth weights}

Owing to high levels of heterogeneity, data for birth weights were not pooled. Two studies reported higher mean birth weights (grams) in the NRT groups $(n=183 ;$ MD $337.0 ; 95 \%$ CI 159.71-514.29 and $n=250$; MD 186.00; 95\% CI $36.00-336.00)$. One study $(n=1042)$ reported a lower mean birth weight in the NRT group (MD $-20.00 ; 95 \%$ CI -27.29 to -12.71 ).

In the one non-placebo-controlled trial $(n=181)$, mean birth weight was lower in the NRT group but it was not significant. (MD $-71.00 ; 95 \%$ CI, -282.13 to 140.13$)$.

Four studies $(n=1628)$ reported data on low birth weights $(<2500 \mathrm{~g})$. Owing to high heterogeneity between three placebo-controlled trials and data from only one non-placebo-controlled trial, a pooled analysis was not conducted and no conclusions could be drawn.

Other outcomes: preterm births (four studies, $n=1628)$, neonatal intensive care unit admissions 
(three studies, $n=1386$ ) and neonatal deaths (three studies, $n=1386)$ ) were lower in NRT groups, but the differences were not significant. Only one study $(n=1024)$ reported a significantly higher caesarean section rate among the NRT group compared with the control (RR 1.36; 95\% CI 1.04-1.77). They reported no significant differences between NRT and placebo groups on other birth outcomes (Apgar score at 5 min after birth, cord arterial blood $\mathrm{pH}$, intraventricular haemorrhage, neonatal convulsions, congenital abnormalities, necrotising enterocolitis, mechanical ventilation of infant, assisted vaginal delivery and maternal death).

\section{Adherence/compliance and side effects}

Adherence reported from five studies was generally low as most participants did not complete the course of NRT. Four trials reported non-serious side effects. Of these, one study reported that $25 \%$ of women in the NRT group experienced minor symptoms, and two women discontinued treatment, but the study did not monitor symptoms in the control group. In another study $10 \%$ of participants experienced headaches, dizziness, fatigue, heartburn, nausea or vomiting, with $15 \%$ in the NRT and $12 \%$ in the control groups unable to continue treatment. One study documented that 11 participants had discontinued patches due to adverse effects (eg, skin irritations and headache) but did not report treatment allocations. Five women in this study also reported palpitations and two reported nausea. One study documented 535 non-serious adverse events reported by 521 participants in the NRT group and 450 reported by the 529 participants in the placebo group.

\section{Implications for practice}

This review shows that NRT is the only pharmacotherapy evaluated in RCTs for smoking cessation in pregnant women. Only six RCTs met the inclusion criteria and there is currently insufficient evidence to establish the effectiveness or safety of NRT when given to pregnant women as an adjunct to behavioural support for smoking cessation. Conclusions on the impact of NRT on birth outcomes can also not be drawn at this stage. Overall, the findings are statistically non-significant so more research is required to establish its effectiveness. Neither varenicline nor bupropion can be recommended for use in pregnancy as no studies evaluating these treatments were identified.

\section{Implications for research}

The dose of NRT varied between studies, although most used a standard dose of NRT, and findings were statistically non-significant. This suggests that research should focus on the dose of NRT as higher doses may be effective and need to be tested in large placebo-controlled RCTs. The reasons for low adherence with NRT need to be examined in qualitative studies exploring experiences of pregnant women in using NRT. Studies of other pharmacotherapies such as bupropion and varenicline are required, if considered to be ethically appropriate.

\section{Acknowledgements}

The author is a member of the Cochrane Nursing Care Field (CNCF).

\section{Financial Support}

This research received no specific grant from any funding agency, commercial or not-for-profit sectors.

\section{Conflicts of Interest}

None.

\section{Ethical Standards}

Not applicable. This is a summary based on secondary research and is not dealing with animals.

\section{References}

National Institute for Health and Clinical Excellence (NICE) Public Health Guidance 26. 2010: Smoking cessation services in primary care, pharmacies, local authorities and workplaces, particularly for manual working groups, pregnant women and hard to reach communities. Retrieved 20 May 2013 from http://www.nice.org.uk/PH010

Coleman, T., Chamberlain, C., Davey, M.A., Cooper, S.E. and Leonardi-Bee, J. 2012: Pharmacological interventions for promoting smoking cessation during pregnancy. Cochrane Database of Systematic Reviews, Issue 9. Art. no.: CD010078. Doi: 10.1002/14651858.CD010078. 